\title{
Metronomic capecitabine as an immune modulator in glioblastoma patients reduces myeloid-derived suppressor cells
}

David M. Peereboom, ${ }^{1,2}$ Tyler J. Alban, ${ }^{3,4}$ Matthew M. Grabowski, ${ }^{1}$ Alvaro G. Alvarado, ${ }^{5}$ Balint Otvos, ${ }^{1}$ Defne Bayik, ${ }^{2,3}$ Gustavo Roversi, ${ }^{3}$ Mary McGraw, ${ }^{1}$ Pengjing Huang, ${ }^{1}$ Alireza M. Mohammadi, ${ }^{1}$ Harley I. Kornblum, ${ }^{5}$ Tomas Radivoyevitch, ${ }^{6}$ Manmeet S. Ahluwalia, ${ }^{1,2}$ Michael A. Vogelbaum, ${ }^{7}$ and Justin D. Lathia ${ }^{1,2,3,4}$

${ }^{1}$ Rose Ella Burkhardt Brain Tumor and Neuro-Oncology Center, Cleveland Clinic, Cleveland, Ohio, USA. ${ }^{2}$ Case Comprehensive Cancer Center, Case Western Reserve University, Cleveland, Ohio, USA. ${ }^{3}$ Cancer Impact Area and Department of Cardiovascular \& Metabolic Sciences, Lerner Research Institute, Cleveland Clinic, Cleveland, Ohio, USA. ${ }^{4}$ Department of Molecular Medicine, Cleveland Clinic Lerner College of Medicine, Case Western Reserve University, Cleveland, Ohio, USA. ${ }^{5}$ Department of Psychiatry and Biobehavioral Sciences and Semel Institute for Neuroscience, University of California, Los Angeles, Los Angeles, California, USA. ${ }^{6}$ Department of Quantitative Health Sciences, Lerner Research Institute, Cleveland Clinic, Cleveland, Ohio, USA. 'Department of NeuroOncology, Moffitt Cancer Center, Tampa, Florida, USA.

BACKGROUND. Myeloid-derived suppressor cells (MDSCs) are elevated in the circulation of patients with glioblastoma (CBM), present in tumor tissue, and associated with poor prognosis. While lowdose chemotherapy reduces MDSCs in preclinical models, the use of this strategy to reduce MDSCs in CBM patients has yet to be evaluated.

METHODS. A phase $0 / I$ dose-escalation clinical trial was conducted in patients with recurrent CBM treated 5-7 days before surgery with low-dose chemotherapy via capecitabine, followed by concomitant low-dose capecitabine and bevacizumab. Clinical outcomes, including progression-free and overall survival, were measured, along with safety and toxicity profiles. Over the treatment time course, circulating MDSC levels were measured by multiparameter flow cytometry, and tumor tissue immune profiles were assessed via time-of-flight mass cytometry.

RESULTS. Eleven patients total were enrolled across escalating dose cohorts of 150, 300 , and 450 $\mathrm{mg}$ bid. No serious adverse events related to the drug combination were observed. Compared with pretreatment baseline, circulating MDSCs were found to be higher after surgery in the $150-\mathrm{mg}$ treatment arm and lower in the 300-mg and 450-mg treatment arms. Increased cytotoxic immune infiltration was observed after low-dose capecitabine compared with untreated CBM patients in the 300-mg and 450-mg treatment arms.

CONCLUSIONS. Low-dose, metronomic capecitabine in combination with bevacizumab was well tolerated in CBM patients and was associated with a reduction in circulating MDSC levels and an increase in cytotoxic immune infiltration into the tumor microenvironment.

TRIAL REGISTRATION. ClinicalTrials.gov NCT02669173.

FUNDING. This research was funded by the Cleveland Clinic, Case Comprehensive Cancer Center, the Musella Foundation, B*CURED, the NIH, the National Cancer Institute, the Sontag Foundation, Blast CBM, the James B. Pendleton Charitable Trust, and the Dr. Miriam and Sheldon C. Adelson Medical Research Foundation. Capecitabine was provided in kind by Mylan Pharmaceuticals.

Copyright: (c) 2019, American Society for Clinical Investigation.

Submitted: June 3, 2019

Accepted: October 3, 2019

Published: November 14, 2019.

Reference information: /CI Insight.

2019;4(22):e130748.

https://doi.org/10.1172/jici.

insight.130748.

\section{Introduction}

Glioblastoma (GBM) is the most common primary malignant brain tumor, with an annual incidence of approximately 11,000 cases in the US and a median survival of 14-18 months despite aggressive therapies (1-5). Currently, standard of care includes maximal safe surgical resection, followed by concomitant 
radiation and chemotherapy with temozolomide (1). In nearly $100 \%$ of cases, this approach fails, resulting in a recurrent tumor with limited therapeutic options. Few therapies are FDA approved for patients with recurrent GBM, including lomustine, bevacizumab, carmustine wafers, and tumor-treating fields, none of which have demonstrated a marked improvement in overall survival (OS) (6-9). Multiple other cancers have faced similar obstacles to effective treatment, but this has been recently overcome with the use of immune-modulating therapies and, as a consequence, there is interest in trying to modify the immune system in GBM. Several immunomodulatory approaches are currently under clinical evaluation, including the use of immune checkpoint inhibitors, oncolytic viruses, dendritic cell vaccines, and CAR-T cell approaches (10), but the well-appreciated immunosuppressive nature of GBM has proven difficult to overcome (11-15).

A hallmark of GBM immunosuppression is the appearance of circulating myeloid-derived suppressor cells (MDSCs) at higher levels than in many other cancers $(11,13,16-20)$. This heterogeneous cell population is activated upon injury and in many cancers, where MDSCs inhibit cytotoxic immune cell populations and contribute to overall immune suppression (21-25). In multiple solid-tumor models and clinical trials, elevated peripheral MDSC levels have correlated with a more immunosuppressive phenotype, as well as with tumors that were refractory to immune-activating therapies, including immune checkpoint inhibitors $(26,27)$. We previously observed that patients with GBM with a better prognosis had reduced MDSCs in their tumors as well as in their peripheral circulation $(13,22)$. Previous studies demonstrated that MDSCs in multiple tumor types can be reduced via low-dose chemotherapies (2830 ). We recently found that this could be achieved in preclinical GBM mouse models via 5-fluorouracil (5-FU), an antimetabolite drug that enters both RNA and DNA; by inhibiting thymidylate synthase and thus lowering dTTP concentrations, dUTP and FdUTP have less competition and are thus more likely to enter DNA $(11,31-33)$. Using a metronomic low-dose 5-FU strategy, we were able to reduce circulating MDSCs, increase intratumoral activated T cell populations, and prolong survival (11). Based on these preclinical observations, we sought to test this approach in recurrent GBM patients using an orally bioavailable 5-FU prodrug, capecitabine, combined with standard-of-care bevacizumab, an antiangiogenic agent. Bevacizumab, an anti-VEGF antibody, was added to ensure that patients received standard therapy for recurrent GBM. In this phase 0/I dose-escalation trial, our objective was to assess the ability of capecitabine to reduce circulating and tumoral MDSCs and to determine the safety/toxicity profile of capecitabine alone and in combination with bevacizumab in this patient population. Based on the hypothesis that this treatment approach would reduce immune suppression, we also analyzed circulating immune cells via flow cytometry and evaluated the immune profile of treated tumors with time-of-flight mass cytometry (CyTOF).

\section{Results}

Phase 0/I clinical trial demographics. Patient accrual began in October 2016. To target MDSCs, we assessed the efficacy of escalating low doses of metronomic capecitabine with a fixed, standard dose of bevacizumab in a clinical trial approved by the Cleveland Clinic Institutional Review Board (ClinicalTrials. gov, NCT02669173). Once GBM recurrence was identified on MRI, study-specific informed consent was obtained. Capecitabine was given 5-7 days before surgery and then continued in 28-day cycles, with periodic blood draws to assess peripheral blood immune cell populations over the course of the trial (Figure 1). A total of 12 patients was enrolled initially, with 1 patient removed and deemed inevaluable after the resection specimen identified pseudoprogression. The demographics of the 11 evaluable patients are summarized in Table 1. The median age at diagnosis was 58 years; 7 patients were enrolled at the time of their first progression, and the remaining 4 patients were enrolled at their second progression. Surgical therapy at diagnosis included biopsy only (2 patients), biopsy with laser ablation (1 patient), and surgical resection (8 patients). All patients then received standard-of-care radiation with concurrent and adjuvant temozolomide. Additional therapies before trial enrollment included other chemotherapies (4 patients: 2 received lomustine and 2 received tesevatinib, a multitargeted tyrosine kinase inhibitor), tumor-treating fields (1 patient), and immunotherapies ( 3 patients). The 3 patients who previously received immunotherapy included 2 patients for whom anti-LAG3 treatment failed and 1 patient who received the SurVaxM vaccine. Five patients had O-6-methylguanine-DNA methyltransferase-methylated GBMs, and one of these had an isocitrate dehydrogenase mutation. Additionally, these 3 prior immunotherapy-treated patients demonstrated no remarkable differences in immune populations compared with the others on the trial. Additional patient details, including molecular markers and other disease-management paradigms, are provided in 


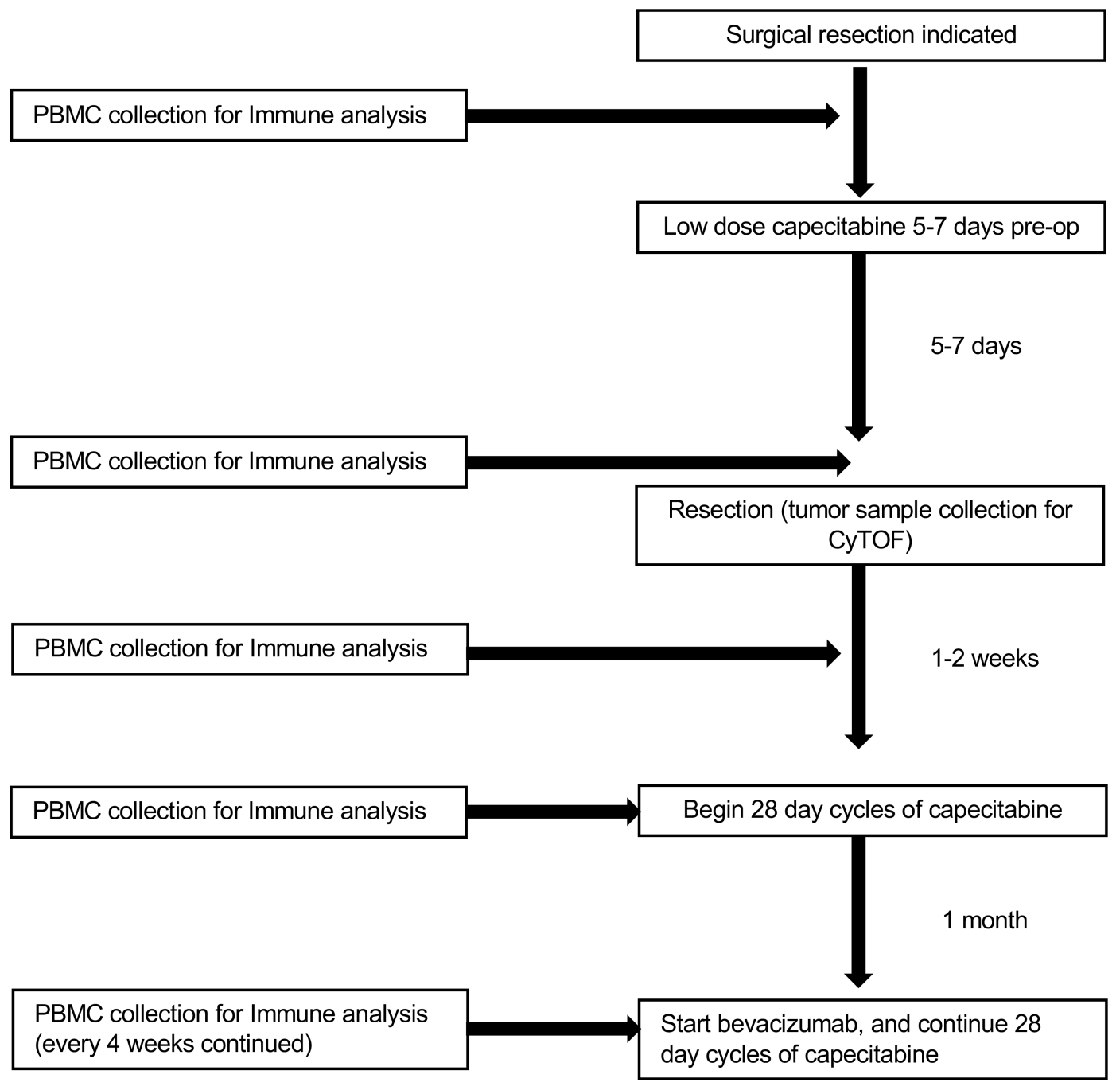

Figure 1. Study schematic demonstrating the time points for capecitabine treatment and immune analysis via flow cytometry and CyTOF.

Supplemental Table 1 (supplemental material available online with this article; https://doi.org/10.1172/ jci.insight.130748DS1). Patients received capecitabine in 3 dose cohorts: $150 \mathrm{mg}$ bid (4 patients), $300 \mathrm{mg}$ bid (3 patients), and $450 \mathrm{mg}$ bid (4 patients).

Tolerability of metronomic low-dose capecitabine therapy in recurrent GBM patients. Capecitabine at all 3 doses administered in combination with bevacizumab was generally well tolerated, with 1 treatment-related (i.e., event possibly, probably, or definitely related to treatment) serious adverse event - grade 5 perforated diverticulum (Table 2). This patient suffered the event after going off study but within 30 days of completion of the study treatment. No other grade 4 or 5 events occurred. Tables 2 and 3 summarize the treatment-related adverse events, which included 1 patient each with grade 3 thromboembolism and dyspnea. One patient in the 450-mg bid cohort experienced grade 3 anemia approximately 2.5 months into the trial, and capecitabine was dose-reduced to $300 \mathrm{mg}$ bid. Other side effects were grade 1 and 2 and included fatigue (4 patients), hypertension ( 3 patients), nausea and vomiting ( 2 patients), and a small intracranial hemorrhage that required no intervention (1 patient).

Daily oral capecitabine reduced the expected increase in MDSCs after surgical resection. Peripheral blood MDSC concentrations were evaluated via flow cytometry (Supplemental Figure 1A) before treatment and during surgical resection and after surgical resection on a per-patient basis to visualize trends in MDSC changes over time (13) (Figure 2A). Relative to each patient's initial value, monitoring MDSCs over time, patients in the 300- and 450-mg bid capecitabine treatment arms had a reduction in MDSCs after surgery that was not observed in the 150-mg bid arm (Figure 2A). In comparison, a longitudinal analysis of newly diagnosed GBM patients conducted in a separate, contemporaneous study showed a 
Table 1. Demographic, clinical, and tumor characteristics of the patients enrolled in the trial

\begin{tabular}{|c|c|}
\hline Variable & Median value (range) \\
\hline Total patients & 11 \\
\hline Age at diagnosis, yr & $58(38-67)$ \\
\hline Male sex & $6 / 11(55 \%)$ \\
\hline Karnofsky performance status & $90(30-100)$ \\
\hline 90 & $7 / 11(64 \%)$ \\
\hline 80 & $4 / 11(36 \%)$ \\
\hline \multicolumn{2}{|l|}{ Ethnicity } \\
\hline White & $11 / 11(100 \%)$ \\
\hline \multicolumn{2}{|l|}{ Molecular markers } \\
\hline $1 p$ loss & $2 / 9(22 \%)$ \\
\hline $19 q$ loss & $2 / 9(22 \%)$ \\
\hline IDH mutated & $1 / 11(9 \%)$ \\
\hline MGMT methylated & $5 / 11(45 \%)$ \\
\hline EGFR amplification & $5 / 9(56 \%)$ \\
\hline \multicolumn{2}{|l|}{ Initial surgical therapy } \\
\hline Biopsy only & $2 / 11(18 \%)$ \\
\hline NTR & $3 / 11(27 \%)$ \\
\hline GTR & $5 / 11(45 \%)$ \\
\hline Laser ablation & $1 / 11(9 \%)$ \\
\hline \multicolumn{2}{|l|}{ Prior local therapy } \\
\hline External beam radiation therapy & $11 / 11(100 \%)$ \\
\hline Tumor treating fields & $1 / 11(9 \%)$ \\
\hline \multicolumn{2}{|l|}{ Prior systemic therapy } \\
\hline $\mathrm{TMZ}$ & $11 / 11(100 \%)$ \\
\hline Immunotherapy & $3 / 11(27 \%)$ \\
\hline Chemotherapy & $4 / 11(36 \%)$ \\
\hline Subsequent chemotherapy & $3 / 11(27 \%)$ \\
\hline \multicolumn{2}{|l|}{ Progression no. at trial enrollment } \\
\hline First & $7 / 11(64 \%)$ \\
\hline Second & $4 / 11(36 \%)$ \\
\hline \multicolumn{2}{|l|}{ Extent of resection } \\
\hline STR & $2 / 11(18 \%)$ \\
\hline NTR & $5 / 11(45 \%)$ \\
\hline GTR & $4 / 11(36 \%)$ \\
\hline \multicolumn{2}{|l|}{ Study dose } \\
\hline 150 mg bid & $4 / 11(36 \%)$ \\
\hline $300 \mathrm{mg}$ bid & $3 / 11(27 \%)$ \\
\hline 450 mg bid & $4 / 11(36 \%)$ \\
\hline \multicolumn{2}{|l|}{ No. of cycles completed } \\
\hline 150 mg bid ${ }^{A}$ & 7.5 cycles $(4-30)$ \\
\hline 300 mg bid & 5 cycles $(2-6)$ \\
\hline 450 mg bid ${ }^{A}$ & 8 cycles (2-14) \\
\hline Overall & 6 cycles $(2-30)$ \\
\hline Progression-free survival at 6 mo. & $5 / 11(45 \%)$ \\
\hline Progression-free survival & $5.8 \mathrm{mo} .(1.8-27.8 \mathrm{mo})$. \\
\hline Overall survival & 11.5 mo. (3.0-28.0 mo.) \\
\hline No. of patients still active on trial at publication ${ }^{B}$ & $2 / 11(18 \%)$ \\
\hline No. of patients still alive at publication ${ }^{\mathrm{B}}$ & $3 / 11(27 \%)$ \\
\hline \multicolumn{2}{|c|}{$\begin{array}{l}\text { Molecular markers for each patient were not available for every test. Percentages are shown of the total patients } \\
\text { who had testing done for that specific marker. IDH, isocitrate dehydrogenase; MGMT, 0-6-methylguanine-DNA } \\
\text { methyltransferase; EGFR, epidermal growth factor receptor; NTR, near-total resection; GTR, gross-total resection; } \\
\text { TMZ, temozolomide; STR, subtotal resection. }{ }^{A} \text { One patient in each group is still active on trial. }{ }^{B} \text { As of May } 1,2019 .\end{array}$} \\
\hline
\end{tabular}


Table 2. Causes of each patient being removed from the study, grouped by capecitabine dose

\begin{tabular}{|c|c|}
\hline Reason off study ( 9 patients ${ }^{A}$ ) & No. (\%) \\
\hline \multicolumn{2}{|l|}{ Dose 1 - 150 mg bid - 4 patients } \\
\hline Radiographic/clinical progression & $2 / 4(50 \%)$ \\
\hline Adverse event/side effects/complications & $1 / 4(25 \%)$ \\
\hline Still receiving study treatment as of 5/1/2019 & $1 / 4(25 \%)$ \\
\hline \multicolumn{2}{|l|}{ Dose 2 - $300 \mathrm{mg}$ bid - 3 patients } \\
\hline Radiographic/clinical progression & $3 / 3(100 \%)$ \\
\hline \multicolumn{2}{|l|}{ Dose $3-450 \mathrm{mg}$ bid - 4 patients } \\
\hline Radiographic/clinical progression & $2 / 4(50 \%)$ \\
\hline Patient withdrawal for other reasons & $1 / 4(25 \%)$ \\
\hline Still receiving study treatment as of 5/1/2019 & $1 / 4(25 \%)$ \\
\hline
\end{tabular}

continued increase in MDSCs over time after surgical resection (Figure 2A). Prior studies suggest that these patients, as well as recurrent GBM patients treated in this trial, undergo spike increases in MDSCs immediately after their surgical resections $(13,34)$. These increases in peripheral blood MDSCs after surgery have been attributed to surgical intervention in multiple cancer types $(16,23,35,36)$, as these increases also occur after surgical resections in untreated GBM patients (Figure 2A). These analyses allowed us to identify a relative reduction in peripheral MDSCs after surgical resection in the 300- and 450-mg bid capecitabine cohorts in 6 of 7 patients (Figure 2A). Of note, patient 3 in the 450-mg bid capecitabine treatment cohort (the only patient who did not have a reduction in peripheral MDSCs after surgery) was noted to have multifocal GBM at the time of recurrence and enrollment in the trial. The distal site of recurrence was not resected, and the patient progressed 1 month later at that lesion site. Therefore, this patient was excluded from additional postsurgical analysis of peripheral blood immune populations. Plotting every single patients' MDSC levels at every time point revealed trends in MDSC changes over time (Supplemental Figure 1B); thus, we decided to analyze MDSCs as fold change compared with each patient's own baseline sample. Relative to each patient's baseline MDSC level, a comparison of the average fold change in MDSCs after surgical resection demonstrated higher levels of MDSCs in untreated patients, a return to preoperative baseline in patients treated with $150 \mathrm{mg}$ bid capecitabine, and a reduction in patients treated with both $300 \mathrm{mg}$ bid and $450 \mathrm{mg}$ bid capecitabine. Statistical analysis using 1-way ANOVA with and without the untreated reference cohort demonstrated significance at $P<$ 0.0001 and $P=0.0308$, respectively. The $300-\mathrm{mg}$ bid capecitabine treatment reduced MDSCs to a level that was not further reduced when the capecitabine treatment was increased to $450 \mathrm{mg}$ bid (Figure 2B). Using a similar flow cytometry approach, analysis of peripheral $\mathrm{T}$ cell populations $\left(\mathrm{CD}^{+}, \mathrm{CD}^{+}, \mathrm{CD}^{+}\right.$, $\mathrm{T}$ regulatory cells) showed no change in the circulation at any dose of capecitabine or in response to surgery (Supplemental Figure 1, B-F).

To further determine the changes in tumor immune profiles associated with systemic capecitabine treatment, we analyzed GBM tissue from patients treated with capecitabine 5-7 days before surgery via CyTOF, which we previously used to identify immune shifts associated with GBM patient prognosis (13). This immune panel consisted of 28 key cell surface immune system markers (Supplemental Figure 2A). In the $\mathrm{CD}_{4} 5^{+}$cell fraction from cryopreserved single-cell tumor suspensions of newly diagnosed GBM patients, a recurrent GBM patient, and GBM patients treated with capecitabine in our trial (300 $\mathrm{mg}$ and $450 \mathrm{mg}$ bid, Figure 3A), we identified 29 immune populations in an unbiased manner using t-distributed stochastic neighbor embedding (tSNE) analyses (Figure 3B and Supplemental Figure 2, $\mathrm{B}$ and $\mathrm{C}$ ). There were no differences in treated versus untreated CD45 cell numbers (Supplemental Figure 2B). Comparing patients with newly diagnosed GBM, recurrent GBM, and recurrent GBM from the capecitabine-dose cohorts, we observed shifts in the tumor-infiltrating immune cell population (Figure 3C and Supplemental Figure 3). Overall, the newly diagnosed and recurrent GBM patients appeared to have similar populations of immune cell clusters, while the groups treated with $300 \mathrm{mg}$ bid and $450 \mathrm{mg}$ capecitabine demonstrated a distinct immune phenotype, resembling a more immune-activated status (Figure 3C and Supplemental Figure 3). Comparing these treated patients with untreated 
Table 3. Treatment-related adverse events that were graded as possibly, probably, or definitely related to the treatment (capecitabine + bevacizumab)

\begin{tabular}{|c|c|c|c|c|c|}
\hline \multirow{2}{*}{$\begin{array}{l}\text { Treatment-related adverse events } \\
\text { ( } n=11 \text { patients) }\end{array}$} & \multicolumn{4}{|c|}{ No. of events by grade } & \multirow[t]{2}{*}{ No. of patients } \\
\hline & Grade 1 & Grade 2 & Grade 3 & Grade 5 & \\
\hline \multicolumn{6}{|l|}{ Hematological adverse events } \\
\hline Thromboembolic event & & & 1 & & $1 / 11(9 \%)$ \\
\hline Thrombocytopenia & & 1 & & & $1 / 11(9 \%)$ \\
\hline Intracranial hemorrhage & & 1 & & & 1/11 (9\%) \\
\hline Peripheral sensory neuropathy & 1 & & & & $1 / 11(9 \%)$ \\
\hline \multicolumn{6}{|l|}{ Gastrointestinal adverse events } \\
\hline Nausea/vomiting & 1 & 1 & & & $2 / 11(18 \%)$ \\
\hline Constipation & 2 & & & & $2 / 11(18 \%)$ \\
\hline \multicolumn{6}{|l|}{ Other adverse events } \\
\hline Fatigue & 2 & 2 & & & $4 / 11(36 \%)$ \\
\hline Hypertension & 2 & 1 & & & $3 / 11(27 \%)$ \\
\hline Dyspnea & 1 & & 1 & & $2 / 11(18 \%)$ \\
\hline Fever & 1 & & & & $1 / 11(9 \%)$ \\
\hline Arthralgia & 1 & & & & $1 / 11(9 \%)$ \\
\hline
\end{tabular}

patients with GBM (including newly diagnosed and recurrent), we observed significant increases in $\mathrm{CD}^{+}$central memory $\mathrm{T}$ cells (subset 1 ), $\mathrm{CD}^{+}$effector memory cells, classical monocytes, dendritic cells, macrophages, microglia (subset 1), NK cells (CD56 $6^{\text {hi }}$ ), and NK cells (CD56 ${ }^{\text {mid }}$ ) (Figure 4 and Supplemental Figure 4, A and B). In addition, the application of a recently published machine-learning algorithm using the $\mathrm{R}$ package CytoDx to the intratumoral CyTOF data revealed that cytotoxic $\mathrm{T}$ lymphocyte-associated antigen 4 (CTLA-4) levels were the most predictive marker to distinguish untreated versus capecitabine-treated patients, and this is represented as a decision tree of predictions showing the predicted cell population changes that occur upon treatment (Figure 5A). Multidimensional (tSNE) analyses of untreated versus capecitabine-treated tumor lymphocytes identified a reduction of CTLA-4 expression (Figure 5B). Manual gating of CyTOF data validated this prediction and identified a significant reduction in CTLA-4 expression in lymphocytes in capecitabine-treated patients (Figure 5C) and also confirmed a significant reduction in CTLA $-4^{+} /$programed cell death protein $1^{+}\left(\mathrm{CTLA}-4^{+} / \mathrm{PD}-1^{+}\right)$ macrophages (Figure 5D). Taken together, these data demonstrate that low-dose systemic capecitabine treatment reduces circulating MDSCs relative to levels when GBMs are resected under standard conditions, with the latter group experiencing a surgery-induced spike. In addition, low-dose capecitabine altered the tumor immune microenvironment, enhancing the number and immunophenotype of cells associated with an antitumor response.

Clinical outcomes. Median follow-up time was 10.7 months (3-29 months). The number of patients still on trial at the time of manuscript submission was 2 of $11(18 \%)$, and the number still alive was $3(27 \%)$. For patients receiving capecitabine bid at 150,300 , and $450 \mathrm{mg}$, respectively, the median progression-free survival (PFS) was 7.2, 5.5, and 7.3 months, and the median OS was 16.6, 11.5, and 9.8 months. It should be noted that survival was not the primary objective of this study, and uncertainties are thus high in regards to these measurements. For example, median PFS for the entire cohort had a $95 \% \mathrm{CI}$ of 1.8 to $>27$ months, and median OS at 11.5 months had a CI of 3.0 to $>28.0$ months. 
A

Longitudinal study identifies a reduction in MDSCs over time at $300 \mathrm{mg}$ bid and $450 \mathrm{mg}$ bid capecitabine

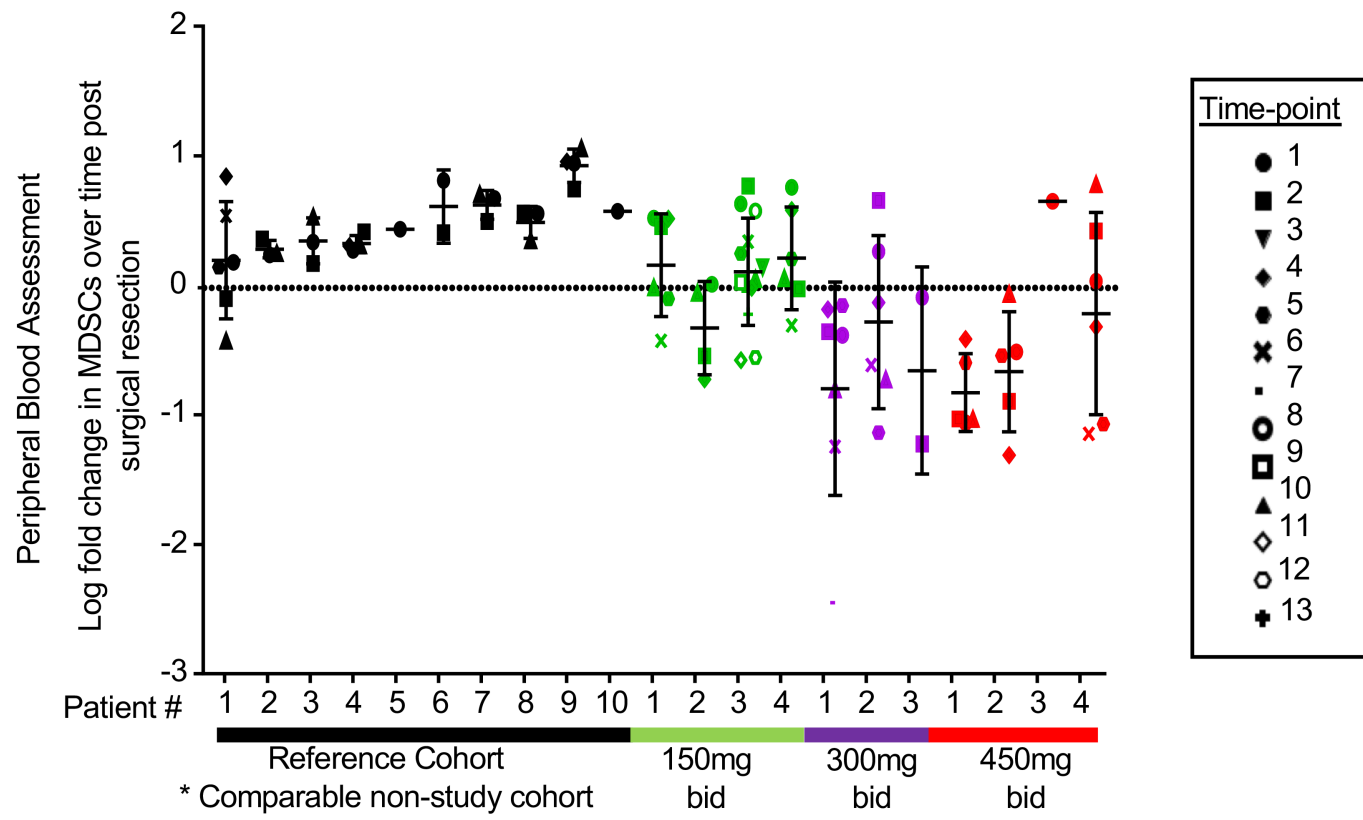

B On average MDSCs are reduced over time in 300mg bid and 450mg bid capecitabine treated patients

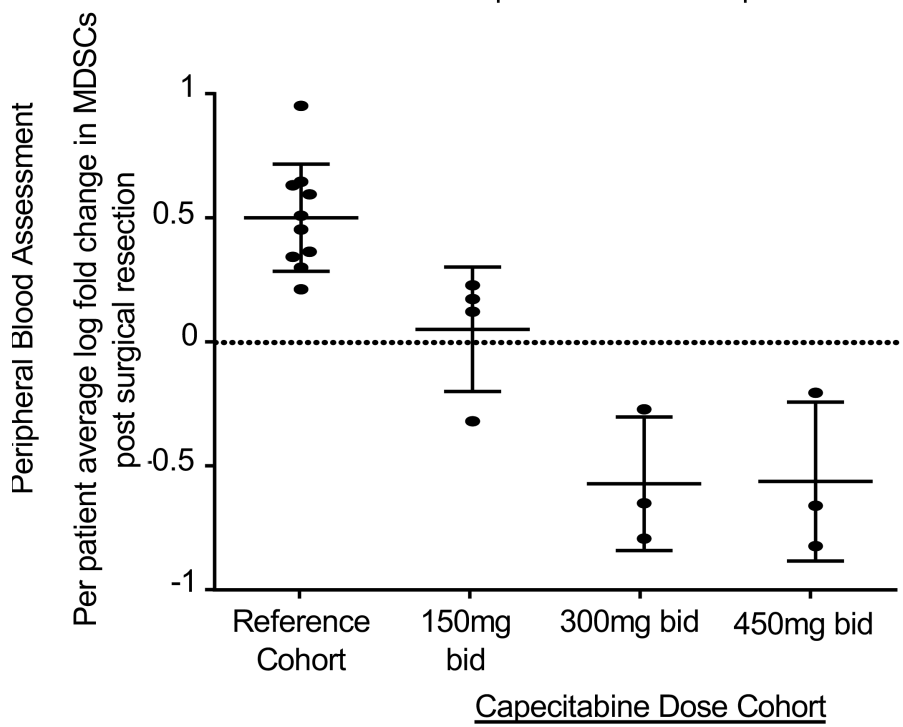

Figure 2. Peripheral MDSCs are reduced over time in patients treated with capecitabine at $\mathbf{3 0 0} \mathbf{~ m g}$ bid and $450 \mathrm{mg}$ bid. Flow cytometry analysis of PBMCs longitudinally identified as MDSCs

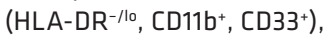
and the log fold change in MDSCs per patient after surgical resection is depicted (A), with each symbol representing the blood draws in sequential order from 1 to 13 ( $n=10$ reference cohort, $n$ $=4$ at $150 \mathrm{mg}$ bid, $n=3$ at 300 $\mathrm{mg}$ bid, $n=4$ at $450 \mathrm{mg}$ bid). The average log fold change of MDSCs per patient over time is graphed per treatment group (B) and identified a significant difference between untreated and all treatment groups and a maximal reduction in the 300-mg bid $(n=3)$ and 450 $\mathrm{mg}$ bid treatment groups ( $n$ = 3) (B). Error bars represent SDs. One-way ANOVA was used to analyze these data; the $F$ test, with and without the reference untreated cohort included as a group, yielded $P<0.0001$ and $P=0.0308$ respectively.

\section{Discussion}

Findings from this study indicate that low-dose capecitabine preoperatively, and postoperatively in combination with bevacizumab, is well tolerated at all doses, with an acceptable side-effect profile. While there is no control arm in this phase 0/I trial, preliminary findings indicate that PFS and OS were at least comparable to historical controls $(1,7)$. Comparisons of the immune populations between the capecitabine-treated groups revealed that $300 \mathrm{mg}$ bid was the optimal capecitabine dose that led to maximal decreases in circulating MDSCs in a subset of patients without affecting lymphoid populations needed for an antitumor immune response. Finally, CyTOF analyses revealed that 5-7 days of priming the immune system with low-dose capecitabine treatment at $300 \mathrm{mg}$ bid and $450 \mathrm{mg}$ bid enhanced the antitumor immune cell populations within the tumor, including $\mathrm{CD}^{+}$effector memory cells and $\mathrm{NK}$ cells. However, these results are underpowered to resolve the complete effects of capecitabine on the 
A

CD45+ clusters of immune cells identified in bulk tumor cells
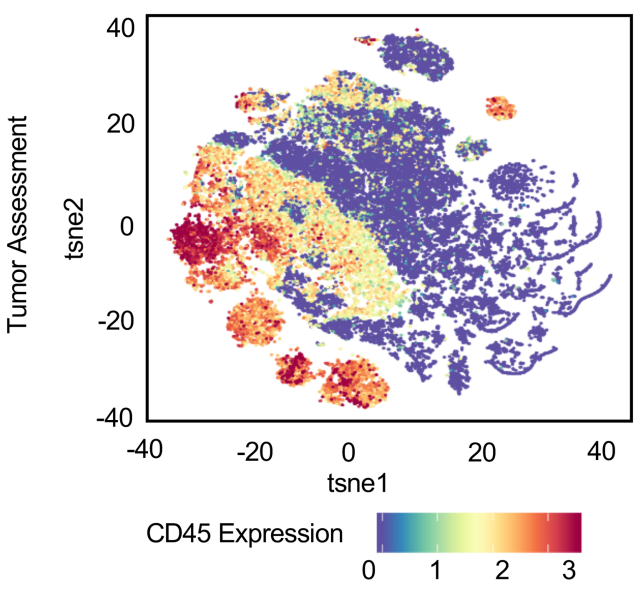

\section{B In depth clustering of CD45+ cells from tumor resections}

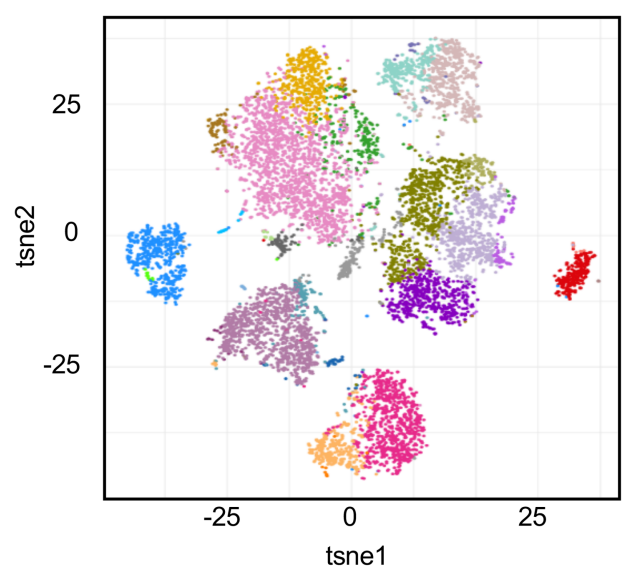

B cells

B cells (CD56 positive)

CD4 Central Memory T cell(subset1)

- CD4 Central Memory T cell(subset2)

- CD4 Effector (Regulatory T cell)

CD4+ Effector Memory

CD8+ Effector (subset1)

CD8+ Effector (subset2)

- CD8+ Effector Memory

- Classical Monocyte

DC

- $\mathrm{DC}(\mathrm{CD} 4+)$

- Double Neg T cells

gMDSCs

- Intermediate Monocyte

Macrophage

- Microlia (subset 1)

- Microglia (subset 2)

- mMDSC

- moDC

Naïve CD4 T cells

- Neutrophil

- NK cells (activated)

NK cells (CD16 high)

- NK cells (CD45 low)

NK cells (CD56 high)

NK cells (CD56 mid)

pDC

- Platelets

C Newly Diagnosed GBM $(n=3) \quad$ Recurrent GBM $(n=1)$
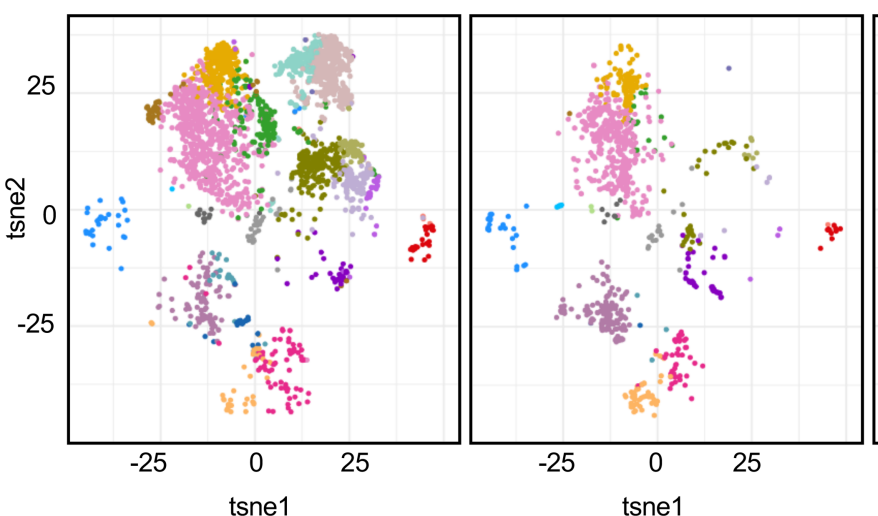

Capecitabine treated $300 \mathrm{mg}$ bid $(\mathrm{n}=2)$
Capecitabine treated $450 \mathrm{mg}$ bid $(\mathrm{n}=3)$

Figure 3. Capecitabine increases the immune activation in tumors after $\mathbf{7}$ days of treatment before surgery. CyTOF analysis using an immune panel of 28 immune markers analyzed capecitabine-treated tumor samples from patients 5, 6, 9, 10, and 11, along with newly diagnosed tumor samples (patients 1, 2, 3), and 1 recurrent GBM tumor (sample 1, $n=4$ total) (A) is represented as a tSNE multidimensional plot and colored by CD45 expression, highlighting the immune populations. After selecting immune populations based on CD45 expression, all tumor sample immune cells were combined and used to cluster immune populations in an unbiased manner from live/CD45 cells only (B). Separate newly diagnosed GBM patient $(n=3)$, recurrent GBM patient $(n=1)$, 300-mg bid capecitabine-treated patient $(n=2)$, and 450-mg bid capecitabine-treated patient $(n=3)$ tSNE plots represent the immune landscape of each tumor cohort (C).

tumor immune responses of the patients; nonetheless, they provide the biological evidence to support the expansion of this strategy to a larger, controlled clinical trial using $300 \mathrm{mg}$ bid capecitabine.

While patients on this trial received both capecitabine and bevacizumab, the tumor immune profile was analyzed on surgical samples obtained before bevacizumab treatment. Therefore, the observed intratumoral effects of capecitabine were not caused by bevacizumab treatment. Furthermore, although circulating MDSC levels were analyzed while patients were on bevacizumab and capecitabine, the noted MDSC changes began to occur before the initiation of bevacizumab. The decreasing MDSC levels also occurred in a capecitabine dose-dependent manner, making it unlikely that bevacizumab played a significant role. However, the potential adjuvant effects of bevacizumab on circulating immune populations cannot be inferred from these results.

In this trial, we used an emerging single-cell phenotyping approach, CyTOF, to gain insight into comprehensive immune signatures within GBM. By integrating these data with a machine-learning approach (37), we identified a potential increase in immune activation after capecitabine treatment. Recently, use 


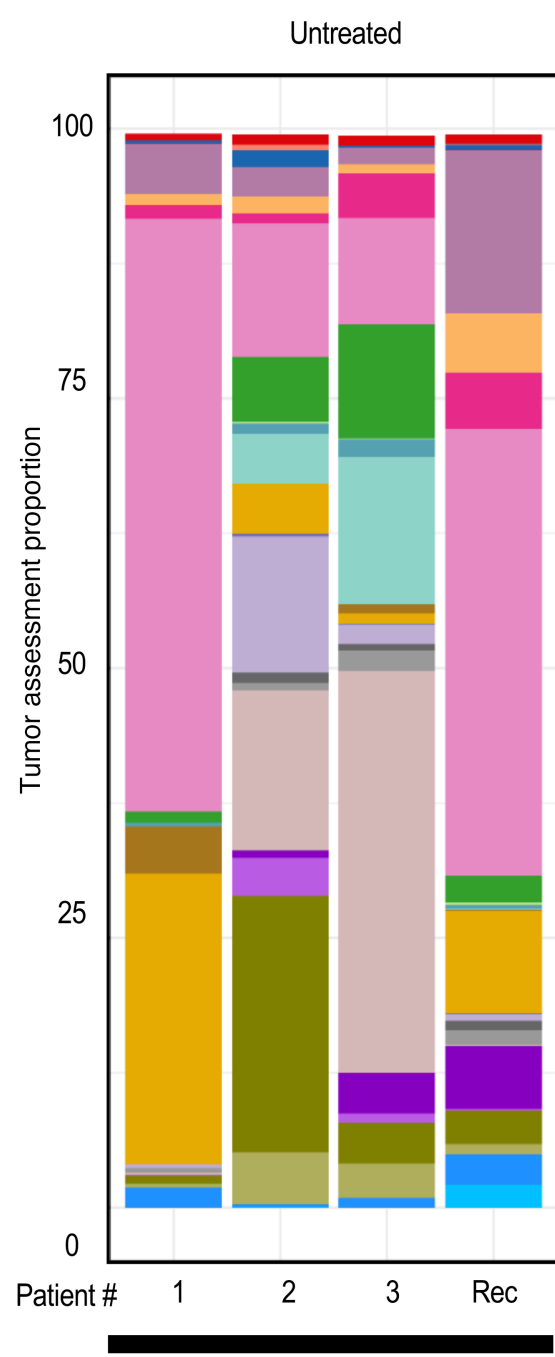

Newly diagnosed
Capecitabine Treated

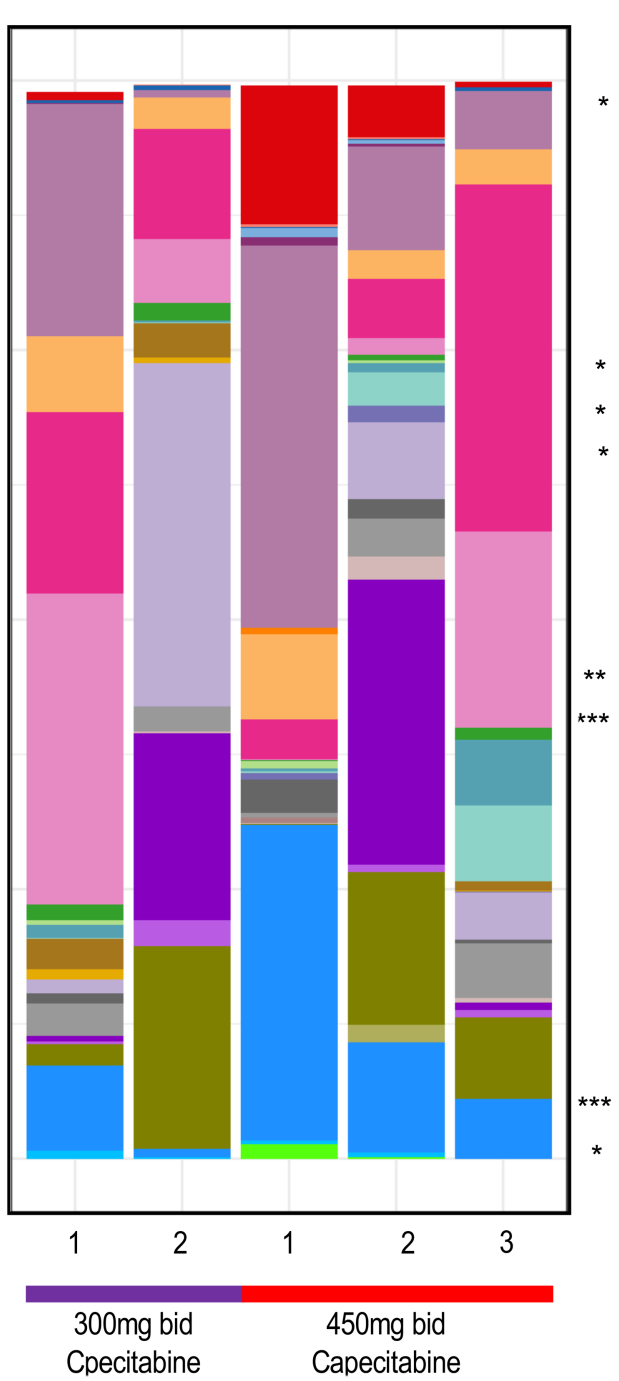

cluster

B cells

B cells (CD56 positive)

CD4 Central memory T cells (subset 1 )

CD4 Central memory $T$ cells (subset 2)

CD4 Effector (regulatory T cells)

CD4+ effector memory

CD8+ effector (subset 1)

CD8+ effector (subset 2)

CD8+ effector memory

Classical Monocyte

DC

DC (CD4+)

Double negative $T$ cells

gMDSC

Intermediate Monocyte

Macrophage

* Microglia (subset 1)

Microglia (subset 2)

mMDSC

$\operatorname{moDC}$

Naiive CD4 T cells

Neutrophil

NK cells (activated)

NK cells (CD16 high)

NK cells (CD45 low)

NK Cells (CD56 high)

NK cells (CD56mid)

pDC

Platelets

Figure 4. Comparison of untreated versus capecitabine-treated immune populations on a per-patient basis. Unbiased clustering identification of immune populations and quantification of the proportion of each cell type present in the $C D 45^{+}$population are represented as a proportion of the total live/CD45 ( $n=1$ patient per bar). Statistical analysis comparing untreated versus treated immune populations identified statistically significant differences between the populations. Linear models of the data with 2-tailed $t$ test comparisons and Benjamini-Hochberg were used to control for multiple comparisons. ${ }^{*} P<0.05,{ }^{* *} P<0.01,{ }^{* *} P<0.001$. Graphs represent data sets as median with first and third quartiles.

of next-generation technology to enhance clinical trials identified key changes between responders and nonresponders (38-40). For example, in 3 recent GBM immunotherapy clinical trials, single-cell RNA-sequencing, CyTOF, and $\mathrm{T}$ cell receptor repertoire/clonality analyses demonstrated changes in the immune microenvironment as a result of therapy, immune alterations as a result of the dynamics of clonal tumor cell evolution, and specific immune signatures associated with response (38-40). Taken together, these approaches demonstrate insight that can be provided by these immune-monitoring approaches. However, it should be noted that this study was designed as a phase 0 trial and, therefore, there are several limitations, including the lack of a control arm and limited statistical power. Our preliminary findings do have the potential to inform a larger-scale clinical trial in the context of specific immune monitoring measurements.

The dosing of chemotherapy agents has historically focused on the maximal tolerated dose to increase antitumor effects. In many cases, these high doses are associated with significant bone marrow toxicity, eliminating both protumor and antitumor immune cells (28, 41-44). However, chemotherapies, such as 5-FU and gemcitabine, differentially effect the immune system at low doses, and this has recently been observed with temozolomide $(12,16,29,45)$. By reducing the dose of these standard 
A

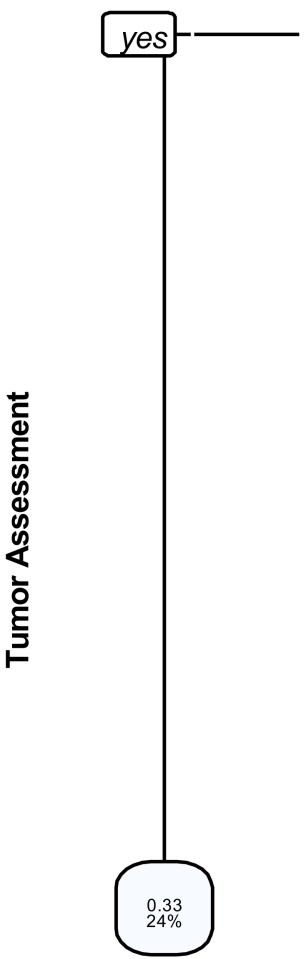

161Dy_CD152_CTLA4

$>=0.067$

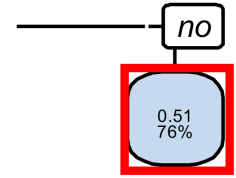

Identifies significant prediction of low

CTLA-4 levels in $76 \%$ of treated patients

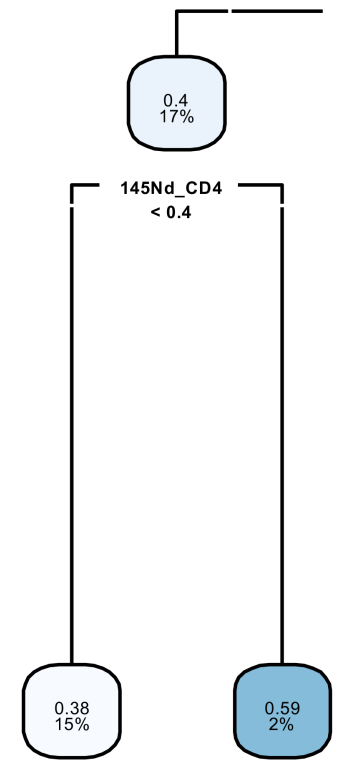

149Sm_CD66a $>=0.041$

patients

B

Untreated $(n=4)$

Capecitabine $(n=5)$
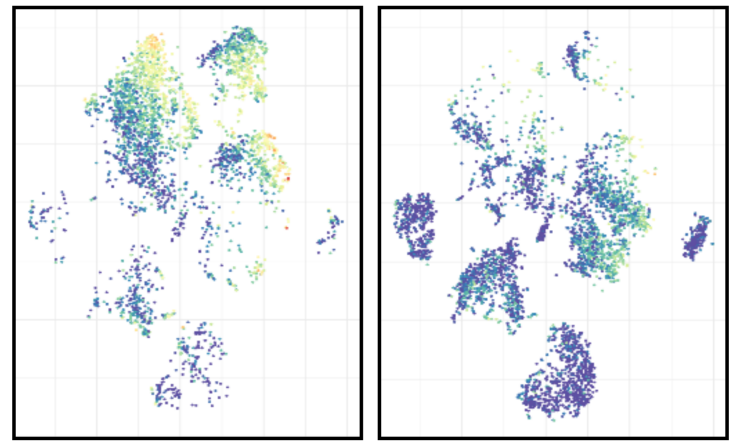

CTLA-4 expression of tumor derived lymphocytes

\section{CTLA4}
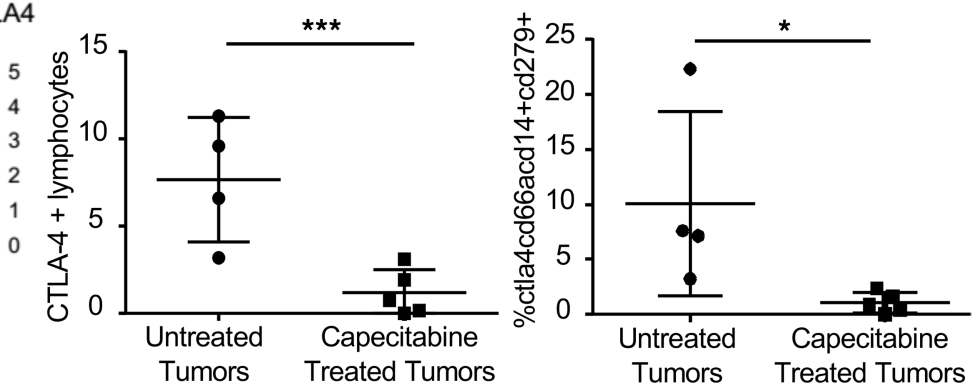

Figure 5. Using patient tumor CyTOF data, a machine-learning approach identified a reduction in a signature for immune cell exhaustion in the tumors of capecitabine-treated patients. From the CyTOF data, a decision tree was generated using the CytoDx R package (A). The first node of the decision tree is highlighted, identifying the initial finding of $76 \%$ of patients with a lower level of CTLA-4 $4^{+}$cells. Multidimensional tSNE modeling of the total CD45 cells from the tumors of untreated and treated patients, colored by CTLA-4 expression levels, identifies the clusters with a reduction in CTLA-4 upon treatment (B). Manual gating of the CyTOF data highlighted the quantitative differences in CTLA-4+ lymphocytes in the tumors of patients treated with capecitabine (C). Further manual gating for the final subset of CTLA-4- cells identified by the decision tree revealed a unique population of $C T L A-4^{+} P D-1^{+}$macrophages that were suppressed upon capecitabine treatment (D) (untreated $n=4$, capecitabine treated $n=5$ ).

chemotherapies, it may be possible to reduce a subset of immune cells that drive immune suppression with little negative effect on antitumor immune cell abundance or function. While the mechanisms of action are not well elucidated, this effect may be due to differential sensitivity and/or different proliferation rates. In this trial, the capecitabine dose ( $300 \mathrm{mg}$ bid) that led to the maximal decrease in peripheral immunosuppressive MDSCs was 4- to 5-fold lower than that used in colorectal cancer (1250 mg bid) (46) and pancreatic cancer (2000 mg bid) (47), and increasing the dose of drug (to 450 $\mathrm{mg}$ bid) did not offer further reductions in immunosuppressive MDSCs. Importantly, these lower doses of capecitabine increased intratumoral immune-activating cell subsets, suggesting that these doses 
were more specific for immunosuppressive compartments. Our previous preclinical models illustrated this strategy of selective MDSC inhibition (11), and with the results of this trial, we validated this approach in humans with recurrent GBM. Combined, these data suggest an optimally administered dosage of $300 \mathrm{mg}$ bid capecitabine, with higher doses yielding no significant gains in MDSC reduction while increasing the propensity for side effects and potential global immune suppression. Future studies should now investigate this approach in newly diagnosed GBM patients at the 300-mg bid dose, based on our observation that newly diagnosed patients have an overall increase in MDSC levels over time (which low-dose capecitabine was demonstrated to reduce, Figure 2A).

This study assessed the paradigm of an immunomodulatory approach based not on activating $\mathrm{T}$ cells but rather on relieving immune suppression in the tumor microenvironment by targeting MDSCs. However, targeting MDSCs as a monotherapeutic strategy may not be sufficient to obtain a durable clinical response. Ongoing approaches are exploring immune-activating strategies in GBM, including immune checkpoint inhibitors, oncolytic viruses, CAR-T cells, and vaccine approaches. Unfortunately, none of these approaches have yet to demonstrate durable immune responses or convincing evidence of patient benefit $(10,48)$. One possible explanation for this lack of clinical benefit is that the treatments tested have yet to overcome the inherent immune-suppressive nature of GBM. Therefore, future studies should be designed to use capecitabine in combination with other immune checkpoint inhibitors, such as anti-PD-1 therapy. This design is based on the observation that, while CTLA-4 was reduced upon treatment, we also observed that PD-1 increased, possibly due to immune activation, in capecitabine-treated patients, making it a practical combinatorial treatment strategy to further enhance the antitumor immune response (Supplemental Figures 5-8). Additionally, the immune checkpoint receptors TIM3 and LAG3 did not appear to be altered upon capecitabine treatment (Supplemental Figures 9 and 10). The immunobiology of GBM is complex, and given the negative results of other clinical immunotherapy trials in GBM, our findings suggest that any successful GBM immunotherapy strategy will likely require a synergistic approach that includes targeting of the immune suppressive/inhibitory effects of MDSCs along with utilization of immune activation strategies to overcome the global GBM-induced immunosuppression phenomenon.

\section{Methods}

\section{Clinical study design}

Clinical trial design. This trial is a phase 0/I study of low-dose capecitabine and bevacizumab in patients with recurrent GBM (ClinicalTrials.gov, NCT02669173). Once GBM recurrence was identified on MRI, study-specific informed consent was obtained. Capecitabine was given 5-7 days before a clinically indicated surgical resection and then continued postoperatively in 28-day cycles with periodic blood draws to assess peripheral blood immune cell populations over the course of the trial (Figure 1). Patients were included if they had a recurrent histologically confirmed WHO grade 4 glioma for which a clinically indicated resection was planned. All subjects were at least 18 years of age, had no prior treatment with capecitabine or bevacizumab, and had a Karnofsky Performance status $\geq 60 \%$. Patients were excluded if they were receiving other investigational agents or if they had a history of adverse reactions to compounds with similar chemical or biologic composition to capecitabine or bevacizumab, active infection with hepatitis B or $\mathrm{C}$ or HIV, or other known malignancy within the past 2 years. To continue on the trial after resection was performed, patients were required to have histologically confirmed tumor recurrence (1 patient with radiation necrosis was removed from the trial at this stage).

The treatment regimen began with capecitabine in the preoperative setting (5-7 days before surgery) with the final preoperative dose the day of surgery. Postoperatively, capecitabine was resumed no sooner than 10 days after surgery after clearance from the surgical team and was given on days 1-28 in 28-day cycles. Bevacizumab was started on cycle 2 of capecitabine in the standard doses of $10 \mathrm{mg} / \mathrm{kg}$ i.v. on days 1 ( \pm 3 days) and 15 ( \pm 3 days) of each cycle. MRI was performed every 8 weeks. Treatment was continued until progressive disease according to RANO (Response Assessment in Neuro-oncology) criteria.

Dose escalation took place with the following doses of capecitabine: $150 \mathrm{mg}$ bid (dose level 1), $300 \mathrm{mg}$ bid (dose level 2), and $450 \mathrm{mg}$ bid (dose level 3). As this trial was for patients with recurrent GBM, bevacizumab was included in addition to the low-dose capecitabine, so that patients also received standard-ofcare therapy (this combination at full doses has been proven to be well tolerated, ref. 49). 
The primary endpoint of the study was the degree of reduction in the concentration of circulating MDSCs. Secondary endpoints included concentration of tissue MDSCs and T regulatory cells in resected GBM, safety and toxicity of continuous low-dose capecitabine alone and with standard dose bevacizumab, PFS at 6 months rate, PFS, and OS.

Specimen collection design. Peripheral blood pharmacodynamic assays were performed at the following time points: (a) baseline (at trial enrollment), (b) upon completion of low-dose oral capecitabine for 5-7 days preoperatively, (c) postoperative day 1, (d) immediately before cycle 1 of postoperative capecitabine, (e) immediately before the addition of bevacizumab (postoperative cycle 2), and (f) every 4 weeks until patient removal from the trial. Tumor tissue was submitted for analysis at the time of surgery. See Supplemental Table 2 for the full study calendar.

As a reference group for correlative studies, a secondary cohort of newly diagnosed GBM patients who were untreated before surgery was followed over time, with peripheral blood pharmacodynamic assays performed at similar time intervals, including on the day of surgery, 2 weeks after surgery, and every 2 months. These patients were the same as those previously analyzed and reported by our group (13). To analyze changes in the tumor microenvironment that occur in response to the 7-day preoperative capecitabine treatment, 3 newly diagnosed GBM tumor samples and 1 recurrent GBM tumor sample were obtained via the Cleveland Clinic Brain Tumor Tissue Bank.

\section{Study assessment}

Clinical outcomes analysis. Data collection and analysis were performed at Cleveland Clinic in May 2019. Demographic, clinical, and molecular pathology characteristics of each patient were obtained from the electronic health record and trial database. PFS was defined as the time from trial enrollment until the diagnosis of progression, and OS was defined as the time from initial histopathologic diagnosis until date of death.

Peripheral blood analysis. Peripheral blood analysis was performed at the Cleveland Clinic between 2017 and 2019, with flow cytometry performed as previously described (13). In brief, peripheral blood mononuclear cells were isolated from whole blood via Ficoll gradient. All samples were processed less than 24 hours after blood draw and then frozen in freezing medium for storage. Staining and analysis were performed using standard protocols previously described, with MDSCs marked by CD11b ${ }^{+}, \mathrm{CD}_{3} 3^{+}$, and HLA-DR ${ }^{-/ \mathrm{lo}}$ and then further subdivided into granulocytic MDSCs $\left(\mathrm{CD} 15^{+}\right)$and monocytic MDSCs $\left(\mathrm{CD} 14^{+}\right)(13,24)$. T regulatory cells were gated as $\mathrm{CD}^{+}, \mathrm{CD}^{+}, \mathrm{CD}^{+} 5^{+}$, and $\mathrm{CD} 127^{-}$, as previously described (13). $\mathrm{CD}^{+} \mathrm{T}$ cells were gated as $\mathrm{CD}^{+}, \mathrm{CD}^{+}$, and $\mathrm{CD}^{-}$. Concentrations of blood MDSCs, immune cells, and relevant secreted factors were measured at baseline, preoperatively and postoperatively, and after the addition of bevacizumab.

Tumor tissue analysis. Analysis of tumor tissue was performed cooperatively at the Cleveland Clinic and University of California, Los Angeles (UCLA) in 2018. Tumor tissue samples were collected during surgery when extra tissue was safely available and was not obtained for all patients. Upon receiving tissue samples, tumors were digested in collagenase IV (STEMCELL Technologies) for 1 hour at $37^{\circ} \mathrm{C}$ before being mechanically dissociated via passage through a $40-\mu \mathrm{M}$ filter. After dissociation, the single-cell suspension was washed in cold RPMI medium before being counted and frozen in freezing medium for future use. Samples were then sent to UCLA, where CyTOF analysis was performed as previously described (13), with the exception that analysis was performed using only the $\mathrm{CD} 45^{+}$fraction of events collected. FCS files were normalized between runs using beads and the Nolan lab normalizer (50). Analyses were not done in a blinded manner. Analysis tools were used in R following the methods described by Nowicka et al. (51)

\section{Statistics}

Baseline demographic and clinical characteristics were characterized by median and range for continuous variables and by frequency distribution and percentage of total for categorical variables. Software used for data processing and analysis included $\mathrm{R}$ (version 3.6.1) via R Studio (version 1.1.463, RStudio Inc.), GraphPad PRISM (version 6, GraphPad Software Inc.), FlowJo (version 10.5.0, FlowJo LLC), and JMP Pro (version 14.0.0, SAS Institute Inc.). Statistical tests included 2-sample, 2-tailed $t$ tests and Wilcoxon rank-sum tests for variables that were, or were approximately (e.g., cell counts), continuous, Pearson's $\chi^{2}$ tests and Fisher's exact test for categorical variables, and 1-way ANOVA $F$ tests for a difference in continuous variables between any pair of groups from a set of more than 2 (adjusting for the testing of multiple pairs). A $P$ value of less than 0.05 was considered significant. The Kaplan-Meier method was used to display times to events. 


\section{Study approval}

This trial is a phase 0/I study of low-dose capecitabine and bevacizumab in patients with recurrent GBM (ClinicalTrials.gov, no. NCT02669173). The clinical trial and retrospective study protocols were approved by the Cleveland Clinic Institutional Review Board (no. 16-085) and the Protocol Review and Monitoring Committee of the Case Comprehensive Cancer Center (CASE7315). All participants provided written informed consent to participate in the clinical trial. The study was conceived, designed, initiated, and performed by the academic investigators. The authors confirm the accuracy and completeness of the data and analysis and the fidelity of the study to the protocol. All authors agreed to submit the manuscript for publication.

\section{Author contributions}

DMP, TJA, MMG, AGA, BO, MAV, and JDL provided conceptualization and design. AMM and MAV performed the surgical collection of treated GBM tissue. TJA, AGA, DB, GR, MM, PH, and MMG performed the experiments. TJA, AGA, MMG, BO, DB, TR, MAV, JDL, MSA, and GR analyzed the data. DMP, TJA, MMG, BO, DB, MAV, and JDL wrote the manuscript. HIK, MAV, and JDL provided financial support. All authors provided final approval of the manuscript.

\section{Acknowledgments}

We thank the patients treated at the Rose Ella Burkhardt Brain Tumor and Neuro-Oncology Center for donation of blood and tumor samples for this study, and we thank the staff of the Rose Ella Burkhardt Brain Tumor and Neuro-Oncology Center for their collaboration in acquiring samples. We thank the Case Comprehensive Cancer Center and the Musella Foundation for their support of this clinical trial. We thank the members of the Lathia laboratory for insightful discussion and constructive comments on the manuscript. We thank Joseph Gerow and Eric Schultz for flow cytometry assistance. We thank the Janis V. Giorgi Flow Cytometry Core Laboratory (JCCC; UCLA) for their assistance with CyTOF experiments. We thank Amanda Mendelsohn and the Center for Medical Art and Photography at the Cleveland Clinic for providing illustrations and Erin Mulkearns-Hubert for editorial assistance. Capecitabine was provided in kind by Mylan Pharmaceuticals. This work was funded by an NIH grant (F31 NS101771 to TJA), a Cancer Biology Training Grant (T32CA059366 to DB), the Sontag Foundation (to JDL), Blast GBM (to JDL and MAV), the Cleveland Clinic VeloSano Bike Race (to JDL and MAV), B*CURED (to JDL and MAV), the Case Comprehensive Cancer Center (to JDL and MAV), and the Cleveland Clinic Brain Tumor Research and Therapeutic Development Research Center of Excellence (to MSA and JDL). Flow cytometry was performed in the UCLA JCCC and Center for AIDS Research Flow Cytometry Core Facility, which is supported by NIH awards P30 CA016042 and 5P30 AI028697. The purchase of the Helios mass cytometer that was used in this work was supported, in part, by the funds provided by the James B. Pendleton Charitable Trust. HIK and AGA were supported by the Dr. Miriam and Sheldon G. Adelson Medical Research Foundation.

Address correspondence to: Justin D. Lathia, Lerner Research Institute, 9500 Euclid Avenue, NC10, Cleveland, Ohio 44195, USA. Phone: 216.445.7475; Email: lathiaj@ccf.org. Or to: Michael A. Vogelbaum, Moffitt Cancer Center, 12902 Magnolia Drive, Tampa, Florida 33612, USA. Phone: 813.745.4251; Email: Michael.Vogelbaum@Moffitt.org.

1. Stupp R, et al. Effects of radiotherapy with concomitant and adjuvant temozolomide versus radiotherapy alone on survival in glioblastoma in a randomised phase III study: 5-year analysis of the EORTC-NCIC trial. Lancet Oncol. 2009;10 (5):459-466.

2. Thakkar JP, et al. Epidemiologic and molecular prognostic review of glioblastoma. Cancer Epidemiol Biomarkers Prev. 2014;23 (10):1985-1996

3. Ostrom QT, Cote DJ, Ascha M, Kruchko C, Barnholtz-Sloan JS. Adult glioma incidence and survival by race or ethnicity in the United States from 2000 to 2014. JAMA Oncol. 2018;4 (9):1254-1262.

4. Koshy M, et al. Improved survival time trends for glioblastoma using the SEER 17 population-based registries. J Neurooncol. 2012;107 (1):207-212.

5. Ostrom QT, Gittleman H, Truitt G, Boscia A, Kruchko C, Barnholtz-Sloan JS. CBTRUS Statistical Report: Primary Brain and Other Central Nervous System Tumors Diagnosed in the United States in 2011-2015. Neuro-oncology. 2018;20 (suppl_4):iv1-iv86.

6. Herrlinger $U$, et al. Lomustine-temozolomide combination therapy versus standard temozolomide therapy in patients with newly diagnosed glioblastoma with methylated MGMT promoter (CeTeG/NOA-09): a randomised, open-label, phase 3 trial. Lancet. 2019;393 (10172):678-688

7. Wick W, et al. Lomustine and bevacizumab in progressive glioblastoma. N Engl J Med. 2017;377 (20):1954-1963.

8. Diaz RJ, Ali S, Qadir MG, De La Fuente MI, Ivan ME, Komotar RJ. The role of bevacizumab in the treatment of glioblastoma. J Neurooncol. 2017;133 (3):455-467. 
9. Gilbert MR, et al. A randomized trial of bevacizumab for newly diagnosed glioblastoma. N Engl J Med. 2014;370 (8):699-708. 10. Lim M, Xia Y, Bettegowda C, Weller M. Current state of immunotherapy for glioblastoma. Nat Rev Clin Oncol. 2018;15 (7):422-442.

11. Otvos B, et al. Cancer stem cell-secreted macrophage migration inhibitory factor stimulates myeloid derived suppressor cell function and facilitates glioblastoma immune evasion. Stem Cells. 2016;34 (8):2026-2039.

12. Authier A, et al. Enhanced immunosuppression by therapy-exposed glioblastoma multiforme tumor cells. Int J Cancer. 2015;136 (11):2566-2578.

13. Alban TJ, et al. Global immune fingerprinting in glioblastoma patient peripheral blood reveals immune-suppression signatures associated with prognosis. JCI Insight. 2018;3 (21):122264.

14. Razavi SM, Lee KE, Jin BE, Aujla PS, Gholamin S, Li G. Immune evasion strategies of glioblastoma. Front Surg. 2016;3:11

15. Jacobs JF, et al. Regulatory T cells and the PD-L1/PD-1 pathway mediate immune suppression in malignant human brain tumors. Neuro-oncology. 2009;11 (4):394-402.

16. Najjar YG, Finke JH. Clinical perspectives on targeting of myeloid derived suppressor cells in the treatment of cancer. Front Oncol. 2013;3:49.

17. Raychaudhuri B, et al. Myeloid derived suppressor cell infiltration of murine and human gliomas is associated with reduction of tumor infiltrating lymphocytes. J Neurooncol. 2015;122 (2):293-301.

18. Raychaudhuri B, et al. Myeloid-derived suppressor cell accumulation and function in patients with newly diagnosed glioblastoma. Neuro-oncology. 2011;13 (6):591-599.

19. Dubinski D, et al. CD4+ T effector memory cell dysfunction is associated with the accumulation of granulocytic myeloid-derived suppressor cells in glioblastoma patients. Neuro-oncology. 2016;18 (6):807-818.

20. Gielen PR, et al. Increase in both CD14-positive and CD15-positive myeloid-derived suppressor cell subpopulations in the blood of patients with glioma but predominance of CD15-positive myeloid-derived suppressor cells in glioma tissue. J Neuropathol Exp Neurol. 2015;74 (5):390-400

21. Highfill SL, et al. Bone marrow myeloid-derived suppressor cells (MDSCs) inhibit graft-versus-host disease (GVHD) via an arginase-1-dependent mechanism that is up-regulated by interleukin-13. Blood. 2010;116 (25):5738-5747.

22. Gielen PR, et al. Elevated levels of polymorphonuclear myeloid-derived suppressor cells in patients with glioblastoma highly express S100A8/9 and arginase and suppress T cell function. Neuro-oncology. 2016;18 (9):1253-1264.

23. Kumar V, Patel S, Tcyganov E, Gabrilovich DI. The nature of myeloid-derived suppressor cells in the tumor microenvironment. Trends Immunol. 2016;37 (3):208-220.

24. Bronte V, et al. Recommendations for myeloid-derived suppressor cell nomenclature and characterization standards. Nat Commun. 2016;7:12150.

25. Youn J, Collazo M, Shalova IN, Biswas SK, Gabrilovich DI. Characterization of the nature of granulocytic myeloid-derived suppressor cells in tumor-bearing mice. J Leukoc Biol. 2012;91 (1):167-181.

26. Larkin J, et al. Combined nivolumab and ipilimumab or monotherapy in untreated melanoma. N Engl J Med. 2015;373 (1):2334. DOI: $10.1056 /$ NEJMoa1504030.

27. Kitano S PM, Cortez C, et al. Myeloid-derived suppressor cell quantity prior to treatment with ipilimumab at $10 \mathrm{mg} / \mathrm{kg}$ to predict for overall survival in patients with metastatic melanoma. Journal of Clinical Oncology. 2012;30 (15_suppl):2518. DOI: 10.1200/jco.2012.30.15_suppl.2518.

28. Shurin MR, Naiditch H, Gutkin DW, Umansky V, Shurin GV. ChemoImmunoModulation: immune regulation by the antineoplastic chemotherapeutic agents. Curr Med Chem. 2012;19 (12):1792-1803.

29. Nars MS, Kaneno R. Immunomodulatory effects of low dose chemotherapy and perspectives of its combination with immunotherapy. Int J Cancer. 2013;132 (11):2471-2478.

30. Vincent J, et al. 5-Fluorouracil selectively kills tumor-associated myeloid-derived suppressor cells resulting in enhanced $\mathrm{T}$ cell-dependent antitumor immunity. Cancer Res. 2010;70 (8):3052-3061.

31. Li LS, et al. DNA mismatch repair (MMR)-dependent 5-fluorouracil cytotoxicity and the potential for new therapeutic targets. Br J Pharmacol. 2009;158 (3):679-692.

32. Blesch KS, Gieschke R, Tsukamoto Y, Reigner BG, Burger HU, Steimer JL. Clinical pharmacokinetic/pharmacodynamic and physiologically based pharmacokinetic modeling in new drug development: the capecitabine experience. Invest New Drugs. 2003;21 (2):195-223.

33. Walko CM, Lindley C. Capecitabine: a review. Clin Ther. 2005;27 (1):23-44.

34. Wang J, et al. Surgery-induced monocytic myeloid-derived suppressor cells expand regulatory T cells in lung cancer. Oncotarget. 2017;8 (10):17050-17058.

35. Ortega-Gómez A, Perretti M, Soehnlein O. Resolution of inflammation: an integrated view. EMBO Mol Med. 2013;5 (5):661-674.

36. Montero AJ, Diaz-Montero CM, Kyriakopoulos CE, Bronte V, Mandruzzato S. Myeloid-derived suppressor cells in cancer patients: a clinical perspective. J Immunother. 2012;35 (2):107-115.

37. Hu Z, Glicksberg BS, Butte AJ. Robust prediction of clinical outcomes using cytometry data. Bioinformatics. 2019;35 (7):1197-1203.

38. Zhao J, et al. Immune and genomic correlates of response to anti-PD-1 immunotherapy in glioblastoma. Nat Med. 2019;25 (3):462-469.

39. Cloughesy TF, et al. Neoadjuvant anti-PD-1 immunotherapy promotes a survival benefit with intratumoral and systemic immune responses in recurrent glioblastoma. Nat Med. 2019;25 (3):477-486.

40. Schalper KA, et al. Neoadjuvant nivolumab modifies the tumor immune microenvironment in resectable glioblastoma. Nat Med. 2019;25 (3):470-476.

41. Kim SK, Demetri GD. Chemotherapy and neutropenia. Hematol Oncol Clin North Am. 1996;10 (2):377-395.

42. Wijayahadi N, Haron MR, Stanslas J, Yusuf Z. Changes in cellular immunity during chemotherapy for primary breast cancer with anthracycline regimens. J Chemother. 2007;19 (6):716-723.

43. Rasmussen L, Arvin A. Chemotherapy-induced immunosuppression. Environ Health Perspect. 1982;43:21-25.

44. Vento S, Cainelli F, Temesgen Z. Lung infections after cancer chemotherapy. Lancet Oncol. 2008;9 (10):982-992.

45. Antonopoulos M, VAN Gool SW, Dionysiou D, Graf N, Stamatakos G. Immune phenotype correlates with survival in patients with GBM treated with standard temozolomide-based therapy and immunotherapy. Anticancer Res. 2019;39 (4):2043-2051.

46. Hirsch BR, Zafar SY. Capecitabine in the management of colorectal cancer. Cancer Manag Res. 2011;3:79-89. 
47. Siddiqui NS, Godara A, Byrne MM, Saif MW. Capecitabine for the treatment of pancreatic cancer. Expert Opin Pharmacother. 2019;20 (4):399-409.

48. Filley AC, Henriquez M, Dey M. Recurrent glioma clinical trial, CheckMate-143: the game is not over yet. Oncotarget. 2017;8 (53):91779-91794.

49. Cunningham D, et al. Capecitabine and oxaliplatin for advanced esophagogastric cancer. N Engl J Med. 2008;358 (1):36-46.

50. Finck R, et al. Normalization of mass cytometry data with bead standards. Cytometry A. 2013;83 (5):483-494.

51. Nowicka M, et al. CyTOF workflow: differential discovery in high-throughput high-dimensional cytometry datasets. F1000Res. 2017;6:748. 